\title{
Bayesian Blind Source Separation for Brain Imaging.
}

\author{
Hichem Snoussi* , Vince D.Calhoun** \\ * IRCCyN, Institut de Recherche en Communications et Cybernétiques de Nantes, \\ Ecole Centrale de Nantes, 44321, Nantes, France, \\ email: snoussi@irccyn.ec-nantes.fr \\ Phone: 00332 40376925, Fax: 0033240376930.
** Olin Neuropsychiatry Research Center, Institute of Living, Hartford, CT 06106, email: vince.calhoun@yale.edu \\ Phone: 1-860-545-7768, Fax: 1-860-545-7797
}

\begin{abstract}
The paper deals with the problem of blind source separation in fMRI data analysis. Our main contribution is to present a maximum likelihood based method to blindly separate the brain activations in an fMRI experiment. Choosing the time frequency domain as the signal representation space, our method relies on the second order statistics and exploits the inter-source diversity. It is efficiently implemented by the EM (ExpectationMaximization) algorithm where the time courses of the brain activations are considered as the hidden variables. The successful separation of the right and left visual cortex activations during a visual fMRI experiment and the extraction of only the relevant tasks corroborate the effectiveness of our proposed separating algorithm.
\end{abstract}

\section{INTRODUCTION}

In this paper, we consider a noisy linear instantaneous mixture:

$$
\boldsymbol{x}_{t}=\boldsymbol{A} \boldsymbol{s}_{t}+\boldsymbol{n}_{t}, t=1, \ldots, T,
$$

where where $\boldsymbol{x}_{t}$ is the $(m \times 1)$ vector of observations at the time $t, \boldsymbol{s}_{t}$ is the $(n \times 1)$ vector of sources, $\boldsymbol{A}$ is the $(m \times n)$ mixing matrix and $\boldsymbol{n}_{t}$ the noise vector assumed to be white and stationary (with unknown covariance $\boldsymbol{R}_{n}$ ). The challenging aspect of the BSS problem is the absence of any exact information about the mixing matrix $\boldsymbol{A}$ (see $[1,2]$ for an overview of the blind source separation problem).

Based on i.i.d source modeling, many proposed algorithms are designed to linearly demix the observations $\boldsymbol{x}_{1 . . T}$. The separation principle in these methods is based on the statistical independence of the reconstructed sources (Independent Component Analysis) [3-7]. However, ICA is designed to efficiently work in the noiseless case. In addition, with the i.i.d assumption, the separation necessarily relies on high order statistics and treating the noisy case with the maximum likelihood approach leads to complicated algorithms [8-10].

Discarding the i.i.d assumption, source separation can be achieved with second order statistics. For instance, second order correlation diversity in the time domain [11],

Corresponding author: Hichem Snoussi. frequency domain [12] or time frequency domain [13] are successfully used to blindly separate the sources. Non stationary second order based methods are also proposed in [14-17]. Stationarity and non stationarity can approximately be seen as dual under Fourier transformation. We have recently proposed a maximum likelihood method to separate noisy mixture of Gaussian stationary sources exploiting this temporal / spectral duality $[18,19]$. The Gaussian model of sources allows an efficient implementation of the EM algorithm [20]. In this contribution, we extend this approach to deal with non stationary sources and a limited sample size of collected observations. Relying on the maximum likelihood principle and the Short Time Fourier Transform (STFT), our approach can be interpreted as a regularized blind identification of the sources spectra. Our method is based on the estimation of the mixing matrix, the sources spectra and the noise covariance matrix. Thus, the same algorithm is applied to overdeterminate and underdeterminate cases without a prewhitening step. The method implicitly incorporates a denoising procedure and it is consequently robust to high level noise. The equations involved in the EM algorithm are very simple to implement. An interesting property of the proposed solution is the exploitation of second order spectral non stationarity. The frequency marginal spectrum (integrating over time the spectrograms) corresponds to an improved version of the separation of noisy stationary sources [18] as the smoothed periodograms, obtained by marginalization, are used instead of the empirical periodograms (corresponding to the Wigner-Ville distribution marginals).

The paper is organized as follows. Section II is devoted to the main contribution of this paper. We develop the EM algorithm implementing the maximum likelihood solution in the time frequency domain. The likelihood criteria is interpreted a regularized matching between the spectral covariances. In Section III, simulations on real fMRI signals illustrate the effectiveness of our proposed method comparing to the ICA solution. 


\section{Regularized Spectral Matching}

The temporal fMRI separation relies on the following mixing model:

$$
\boldsymbol{X}=\boldsymbol{A} \boldsymbol{S}+\boldsymbol{N}
$$

where $\boldsymbol{X}$ is the $(M \times T)$ matrix of observations, the column $\boldsymbol{X}(:, t)$ contains the scanned image acquired at the time $t$ and $M$ is the number of voxels in one brain slice. The $(N \times T)$-matrix $\boldsymbol{S}$ (the sources) contains the $N$ time courses rows. The $(M \times N)$ mixing matrix $\boldsymbol{A}$ contains the $N$ brain region activations. Each column $(M \times 1)$ of $\boldsymbol{A}$ represents a source image and it is time invariant. Thus, the column $\boldsymbol{S}(:, t)$ represents the linear combination at the instant $t$ to produce the image $\boldsymbol{X}(:, t)$ (See Figure 1 for illustration). The matrix $\boldsymbol{N}$ models the noise corrupting the observations. The advantage of taking into account the noise in the model is to allow the separating algorithm to only extract the relevant sources, that is the event related brain activations. This is possible when the spectral profile of the noise is flat comparing to the more concentrated source spectra. In fact, the time courses of event related activations present the same frequency content as the stimulus.

Some of the real signals collected in fMRI imaging are obviously non stationary. However, the time courses corresponding to regions which activations are event related are approximately stationary. In addition, their spectral behavior encloses the signature of the stimulus presented to the subject during scanning. The difficulties thus arising when separating the different temporal brain activations are the following:

1. The observations are mixture of two types of sources: stationary sources (event related activations, thermal noise,...) and non stationary sources (artifacts).

2. The moderate time duration of scanning within the limited spectral information provided by time courses make the blind mixing identification a difficult task. In fact, the success of blind separation relies on a good spectral estimation since it is based on structuring the observation covariance matrices according to the linear mixing model. 3 . The noise $\boldsymbol{N}$ is not white and especially when the observation model is modified to segregate task related signals from non task related signals, that is the observation process is written as,

$$
\boldsymbol{X}=\boldsymbol{A}_{t r c} \boldsymbol{S}_{t r c}+\boldsymbol{A}_{a r t} \boldsymbol{S}_{a r t}+\boldsymbol{N}
$$

where the subscript "trc" refers to task related component and the subscript "art" refers to artifact components. Thus, the effective noise $\tilde{\boldsymbol{N}}$ is a composition $\boldsymbol{A}_{\text {art }} \boldsymbol{S}_{\text {art }}+\boldsymbol{N}$ of mixed artifacts and physical noise. It yields a spatial structure due the matrix $\boldsymbol{A}_{\text {art }}$ and a time correlation due to presence of artifacts $\boldsymbol{S}_{\text {art }}$.

4. The sources, in particular the event related ones, are correlated. Therefore, the basic assumption in ICA is no more valid as the sources are not statistically independent. This fact corroborates the use of the Bayesian approach where one could take into account such correlation in the

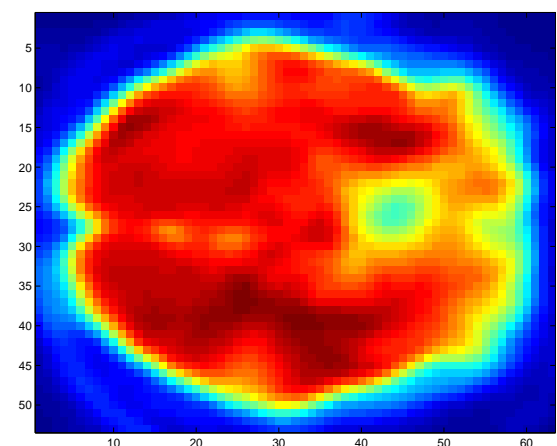

(a)
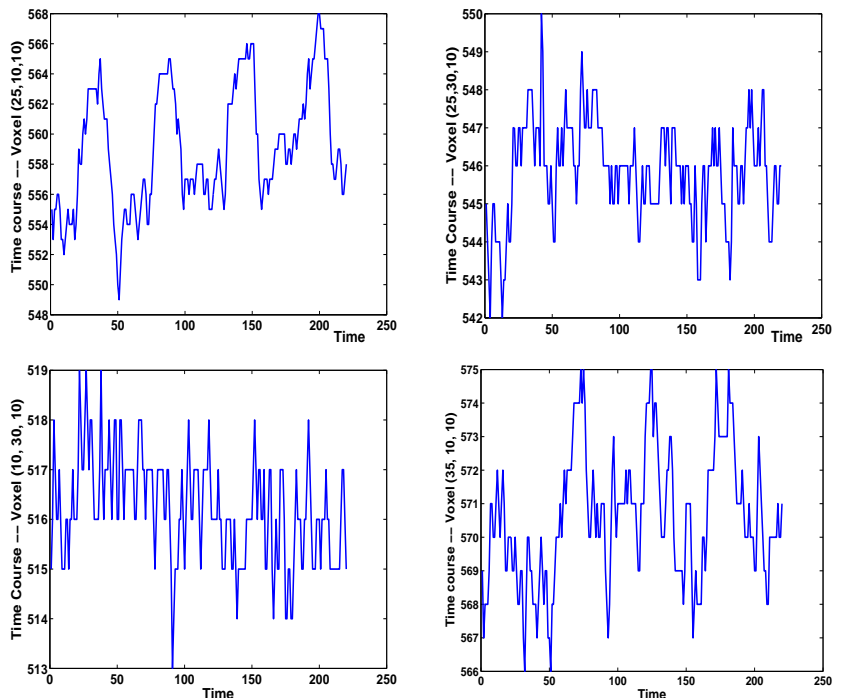

(b)

Fig. 1. Example of an fMRI experiment: (a) the image scanned at the time $t=5 \mathrm{~s}$, it is represented by the column $\boldsymbol{X}(:, t=5)$, (b) Time courses of four different voxels located at $\nu_{1}=(25,10,10), \nu_{2}=$ $(25,3010), \nu_{3}=(10,30,10)$ and $\nu_{4}=(35,10,10)$, they are represented by the rows of the matrix $\boldsymbol{X}: \boldsymbol{X}\left(\nu_{1},:\right), \boldsymbol{X}\left(\nu_{2},:\right), \boldsymbol{X}\left(\nu_{3},:\right)$, and $\boldsymbol{X}\left(\nu_{4},:\right)$

source covariance matrix $\boldsymbol{P}_{s}$ by introducing non null extra diagonal elements.

In the following, we outline the proposed EM algorithm called hereafter the "Regularized Spectral EM" as it is the extension of the Spectral EM [18] and show some separation results on noisy real fMRI data.

The Short Time Fourier Transform (STFT) of a signal $\{x(t)\}$ is a windowed Fourier transform defined as:

$$
S_{x}(t, \omega)=\int x(\tau) h(\tau-t) e^{-j \omega \tau} d \tau
$$

where $h$ is the moving window capturing the signal non stationarity. It is shown that the squared modulus of $S_{x}$ (called the spectrogram) belongs to the Cohen's Class with the kernel $\phi$ equal to $W_{h}$, the Wigner-Ville distribution of the window $h$. Thus, the spectrogram enjoys the positivity property but does not conserve the marginal properties of the Wigner-Ville distribution.

Exploiting the linearity of the STFT transform, the noisy linear mixture model conserves its algebraic form under 
this transformation:

$$
\boldsymbol{x}(t, \omega)=\boldsymbol{A s}(t, \omega)+\boldsymbol{n}(t, \omega), t=1 . . T, \omega=1 . . F,
$$

where, for the sake of clarity, $\boldsymbol{x}, \boldsymbol{s}$ and $\boldsymbol{n}$ also denote the STFT transforms of the observations, the sources and the noise respectively. Assuming that the noise is stationary white (with unknown covariance $\boldsymbol{R}_{n}$ ) and that the sources are decorrelated in the time frequency domain $^{1}$ (with unknown diagonal covariances $\{\boldsymbol{P}(t, \omega)=$ $\left.\mathrm{E}[\boldsymbol{s}(t, \omega) \boldsymbol{s}(t, \omega) *]\}_{t=1 . . . T}^{\omega=1 . F}\right)$, the likelihood is as follows:

$$
\begin{aligned}
& \mathrm{p}(\boldsymbol{X} \mid \boldsymbol{\theta})=\int \mathrm{p}\left(\boldsymbol{X} \mid \boldsymbol{S}, \boldsymbol{A}, \boldsymbol{R}_{n}\right) \mathrm{p}(\boldsymbol{S} \mid\{\boldsymbol{P}(t, \omega)\}) d \boldsymbol{S} \\
& =\prod_{t, \omega} \int \mathrm{p}\left(\boldsymbol{x}(t, \omega) \mid \boldsymbol{s}(t, \omega), \boldsymbol{A}, \boldsymbol{R}_{n}\right) \mathrm{p}(\boldsymbol{s}(t, \omega) \mid \boldsymbol{P}(t, \omega)) d \boldsymbol{s}(t, \omega) \\
& =\prod_{t, \omega}\left|2 \pi \boldsymbol{R}_{t, \omega}\right|^{-1} \exp \left[-\operatorname{Tr}\left(\boldsymbol{R}_{t, \omega}^{-1} \boldsymbol{x}(t, \omega) \boldsymbol{x}(t, \omega)^{*}\right)\right]
\end{aligned}
$$

where $\boldsymbol{R}_{t, \omega}=\boldsymbol{A} \boldsymbol{P}_{t, \omega} \boldsymbol{A}^{*}+\boldsymbol{R}_{n}$ and $\boldsymbol{\theta}$ is the whole parameter to be estimated $\left(\boldsymbol{A}, \boldsymbol{R}_{n},\{\boldsymbol{P}(t, \omega)\}\right)$.

The likelihood (1) can be interpreted as the matching between STFT covariances matrices $\boldsymbol{R}_{t, \omega}=\boldsymbol{A} \boldsymbol{P}_{t, \omega} \boldsymbol{A}^{*}+\boldsymbol{R}_{n}$ and empirical covariances $\hat{\boldsymbol{R}}_{t, \omega}=\boldsymbol{x}(t, \omega) \boldsymbol{x}(t, \omega)^{*}$, in the Kullback-Leibler metric:

$$
\log \mathrm{p}(\boldsymbol{X} \mid \boldsymbol{\theta})=-\sum_{t, \omega} D_{K L}\left(\boldsymbol{R}_{t, \omega} l \hat{\boldsymbol{R}}_{t, \omega}\right)
$$

\section{A. Time frequency EM algorithm}

The first step of the EM algorithm is the computation of the functional $\mathcal{Q}\left(\boldsymbol{\theta}, \boldsymbol{\theta}^{(m-1)}\right)$ :

$$
\begin{aligned}
& \mathcal{Q}\left(\boldsymbol{\theta}, \boldsymbol{\theta}^{(m-1)}\right)=\mathrm{E}\left[\log (\boldsymbol{X}, \boldsymbol{S} \mid \boldsymbol{\theta}) \mid \boldsymbol{X}, \boldsymbol{\theta}^{(m-1)}\right] \\
& =\mathrm{E}\left[\sum_{t, \omega}-\log \left|\boldsymbol{R}_{n}\right|-\operatorname{Tr}\left(\boldsymbol{R}_{n}^{-1}\left[\boldsymbol{x}_{t, \omega}-\boldsymbol{A} \boldsymbol{s}_{t, \omega}\right]\left[\boldsymbol{x}_{t, \omega}-\boldsymbol{A} \boldsymbol{s}_{t, \omega}\right]^{*}\right)\right. \\
& \left.+\sum_{t, \omega}-\log \left|\boldsymbol{P}_{s}(t, \omega)\right|-\operatorname{Tr}\left(\boldsymbol{P}_{s}^{-1}(t, \omega)\left[\boldsymbol{s}_{t, \omega}\right]\left[\boldsymbol{s}_{t, \omega}\right]^{*}\right)\right]
\end{aligned}
$$

Defining the following statistics which will be computed later:

$$
\left\{\begin{array}{l}
\boldsymbol{R}_{x x}(t, \omega)=\boldsymbol{x}_{t, \omega} \boldsymbol{x}_{t, \omega}^{*} \\
\boldsymbol{R}_{x s}(t, \omega)=\boldsymbol{x}_{t, \omega} \mathrm{E}\left[\boldsymbol{s}_{t, \omega} \mid \boldsymbol{x}_{t, \omega}, \boldsymbol{\theta}^{(m-1)}\right]^{*} \\
\boldsymbol{R}_{s s}(t, \omega)=\mathrm{E}\left[\boldsymbol{s}_{t, \omega} \boldsymbol{s}_{t, \omega}^{*} \mid \boldsymbol{x}_{t, \omega}, \boldsymbol{\theta}^{(m-1)}\right]
\end{array}\right.
$$

\footnotetext{
${ }^{1}$ The decorrelation assumption of the time frequency source points is only statistically valid for underspread signals, i.e. the ambiguity function is concentrated in a small neighborhood of the origin [21]. However, our main objective is the estimation of the unknown parameters and not the filtering of sources.
}

the functional $\mathcal{Q}$ can be rewritten in the following form:

$$
\begin{aligned}
& \mathcal{Q}\left(\boldsymbol{\theta}, \boldsymbol{\theta}^{(m-1)}\right)=\sum_{t, \omega}-\log \left|\boldsymbol{R}_{n}\right|-\operatorname{Tr}\left(\boldsymbol { R } _ { n } ^ { - 1 } \left[\boldsymbol{R}_{x x}(t, \omega)\right.\right. \\
& \left.\left.+\boldsymbol{A} \boldsymbol{R}_{s s}(t, \omega) \boldsymbol{A}^{*}-\boldsymbol{A} \boldsymbol{R}_{s x}(t, \omega)-\boldsymbol{R}_{s x}^{*}(t, \omega) \boldsymbol{A}^{*}\right]\right) \\
& +\sum_{t, \omega}-\log \left|\boldsymbol{P}_{s}(t, \omega)\right|-\operatorname{Tr}\left(\boldsymbol{P}_{s}^{-1}(t, \omega) \boldsymbol{R}_{s s}(t, \omega)\right)
\end{aligned}
$$

The second step is the update of the parameter $\boldsymbol{\theta}$ by maximizing the functional $\mathcal{Q}\left(\boldsymbol{\theta}, \boldsymbol{\theta}^{(m-1)}\right)$ :

$$
\boldsymbol{\theta}^{(m)}=\underset{\boldsymbol{\theta}}{\arg \max } \mathcal{Q}\left(\boldsymbol{\theta}, \boldsymbol{\theta}^{(m-1)}\right)
$$

This can be achieved by differentiating the functional $\mathcal{Q}$ (4) with respect to the parameter $\boldsymbol{\theta}$ and then equating to zero the partial derivatives. We obtain the following simple updating equations:

$$
\left\{\begin{array}{l}
\boldsymbol{A}^{(m)}=\boldsymbol{R}_{x s} \boldsymbol{R}_{s s}^{-1} \\
\boldsymbol{R}_{n}^{(m)}=\boldsymbol{R}_{x x}-\boldsymbol{R}_{x s} \boldsymbol{R}_{s s}^{-1} \boldsymbol{R}_{s x} \\
\boldsymbol{P}_{s}(t, \omega)=\operatorname{diag}\left(\boldsymbol{R}_{s s}(t, \omega)\right)
\end{array}\right.
$$

where the matrices $\boldsymbol{R}_{x x}, \boldsymbol{R}_{x s}$ and $\boldsymbol{R}_{s s}$ are the average of the statistic matrices $\boldsymbol{R}_{x x}(t, \omega), \boldsymbol{R}_{x s}(t, \omega)$ and $\boldsymbol{R}_{s s}(t, \omega)$ defined in (3), over the time frequency domain.

The computation of the statistic matrices (3) is essentially based on the computation of the a posteriori first and second moments of the source vector $s_{t, \omega}$. Thanks to the a priori Gaussianity of sources and noise, the a posteriori distribution of the sources is also Gaussian with the following moments:

$$
\left\{\begin{array}{l}
\mathrm{E}\left[\boldsymbol{s}_{t, \omega}\right] \\
\mathrm{E}\left[\boldsymbol{s}_{t, \omega} \boldsymbol{s}_{t, \omega}\right]=\boldsymbol{W}_{t, \omega} \boldsymbol{x}_{t, \omega} \\
\boldsymbol{V}_{t, \omega}+\mathrm{E}\left[\boldsymbol{s}_{t, \omega}\right] \mathrm{E}\left[\boldsymbol{s}_{t, \omega}\right]^{*}
\end{array}\right.
$$

where the matrices $\boldsymbol{W}_{t, \omega}$ (Wiener matrix) and $\boldsymbol{V}_{t, \omega}$ (a posteriori covariance) have the following expressions:

$$
\left\{\begin{array}{l}
\boldsymbol{W}_{t, \omega}=\left[\boldsymbol{A}^{*} \boldsymbol{R}_{n}^{-1} \boldsymbol{A}+\boldsymbol{P}_{s}^{-1}(t, \omega)\right]^{-1} \boldsymbol{A}^{*} \boldsymbol{R}_{n}^{-1} \\
\boldsymbol{V}_{t, \omega}=\left[\boldsymbol{A}^{*} \boldsymbol{R}_{n}^{-1} \boldsymbol{A}+\boldsymbol{P}_{s}^{-1}(t, \omega)\right]^{-1}
\end{array}\right.
$$

We note that the equations are very similar to a time frequency Wiener filtering. Consequently, the EM algorithm involves an implicit denoising procedure when computing the first a posteriori moment of the sources. In other words, we have an optimal source reconstruction at each step of the algorithm. It is worth noting that the achievement of the separation solution is strongly linked to the diversity of the sources spectrograms (the diagonal time frequency distributions of the matrices $\boldsymbol{P}(t, w)$ are different). This is the fundamental reason to perform the separation in the frequency domain when the only temporal statistics are not able to provide such diversity.

\section{B. Spectrum Regularization}

The estimation of the parameter $\boldsymbol{\theta}$ involves the estimation of the whole spectrograms $\left(\left\{\boldsymbol{P}_{s}(t, \omega)\right\}_{t=1 . . T}^{\omega=1 . . F}\right)$ which 
are smoothed versions of the Wigner-Ville spectra. In order to accelerate the EM algorithm, we can partition the time frequency domain into $L$ horizontal sub-domains $\left\{\mathcal{D}_{l}\right\}_{l=1}^{L}$ and then estimate the averaged spectrograms inside these domains. This is algorithmically equivalent to assume that the spectrograms are constant in the sub-domains in the partitioned time frequency 2-D field. Figure 2 illustrate the horizontal segmentation of the time frequency domain.

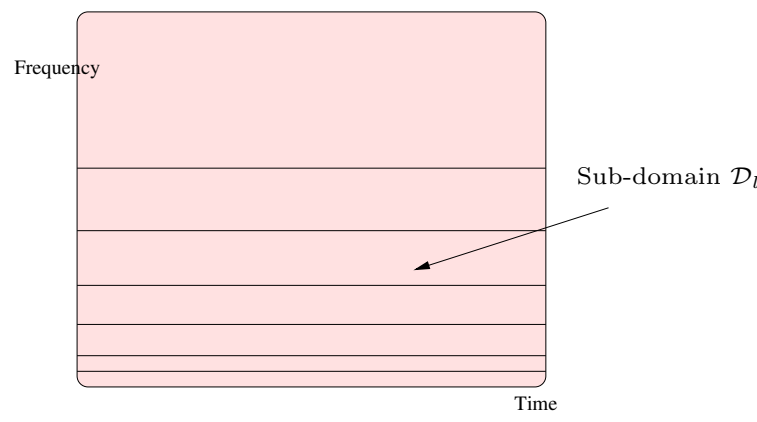

Fig. 2. Marginal partitioning of the Time Frequency domain: exploitation of the spectral non stationarity.

In the following, we show how the expressions of the updating equations of the EM algorithms are very suitable for such approximation.

We assume that $\boldsymbol{P}_{s}(t, \omega)=\boldsymbol{P}_{l}$ for all $(t, \omega) \in \mathcal{D}_{l}$ and the likelihood function can be rewritten as follows:

$\mathrm{p}(\boldsymbol{X} \mid \boldsymbol{\theta})=\prod_{l=1}^{L}\left|2 \pi \boldsymbol{R}_{l}\right|^{-w_{l}} \exp -\operatorname{Tr}\left(\boldsymbol{R}_{l}^{-1} \sum_{(t, \omega) \in \mathcal{D}_{l}} \boldsymbol{x}_{t, \omega} \boldsymbol{x}_{t, \omega}^{*}\right)$

where $w_{l}=\left|\mathcal{D}_{l}\right|$ and $\boldsymbol{R}_{l}=\boldsymbol{A} \boldsymbol{P}_{l} \boldsymbol{A}^{*}+\boldsymbol{R}_{n}$ which is constant in the sub-domain $\mathcal{D}_{l}$.

As the spectral coefficients are constant in each subinterval $\mathcal{D}_{l}$, the statistics are easily computed. In fact, the matrices $\boldsymbol{V}_{t, \omega}=\boldsymbol{V}_{l}$ and $\boldsymbol{W}_{t, \omega}=\boldsymbol{W}_{l}$ are constant over each sub-domain $\mathcal{D}_{l}$ and the statistics are:

$$
\left\{\begin{aligned}
\boldsymbol{R}_{x x}(l) & =\frac{1}{w_{l}} \sum_{(t, \omega) \in \mathcal{D}_{l}} \boldsymbol{x}_{t, \omega} \boldsymbol{x}_{t, \omega}^{*} \longrightarrow \text { computed off line } \\
\boldsymbol{R}_{x s}(l) & =\boldsymbol{R}_{x x}(l) \boldsymbol{W}_{l}^{*} \\
\boldsymbol{R}_{s s}(l) & =\boldsymbol{W}_{l} \boldsymbol{R}_{x x}(l) \boldsymbol{W}_{l}^{*}+\boldsymbol{V}_{l}
\end{aligned}\right.
$$

Then, the statistic matrices $\boldsymbol{R}_{x x}, \boldsymbol{R}_{x s}$ and $\boldsymbol{R}_{s s}$ are the weighted sums of $\boldsymbol{R}_{x x}(l), \boldsymbol{R}_{x s}(l)$ and $\boldsymbol{R}_{s s}(l)$ (with weights $\left.\left\{w_{l}\right\}_{l=1 . . L}\right)$ and both the mixing matrix $\boldsymbol{A}$ and the noise covariance $\boldsymbol{R}_{n}$ are still updated according to the equation (5). The sources spectrograms are updated according to the following equation:

$$
\boldsymbol{P}_{l}=\operatorname{diag}\left(\boldsymbol{R}_{s s}(l)\right)
$$

Remark 1: An interesting property of the EM algorithm is the fact that the computation of the statistic matrices $\boldsymbol{R}_{x s}(l)$ and $\boldsymbol{R}_{s s}(l)$ relies on an off-line computation of the observations spectrograms $\boldsymbol{R}_{x x}(l)$. The computation of the a posteriori expectation of sources is no more necessary leading to a fast implementation of the EM algorithm.

Partitioning the time frequency domain into horizontal bands sub-domains $\left\{\mathcal{D}_{l}\right\}$, the matching of STFT spectra leads to the same algorithm as in [22] exploiting the temporal stationarity but with regularized spectra. In fact, the projection of the STFT spectrum yields the windowed power spectrum. In Figure 3, we have plotted an fMRI time course in time, its spectrum, its STFT transform and the windowed periodogram (projection of the spectrogram) illustrating the effect of smoothing the spectrum of an fMRI time course. Maximizing the likelihood is then equivalent to matching the windowed periodograms according to equation (2). Thus, the method will essentially consist in maximizing the likelihood of the parameters based on the Gaussian modeling of the sources.

As the computation of the observations spectra is performed off-line, the structure of the algorithm is independent of the partition choice. In fact, the algorithm is only based on matching the computed matrices to structured matrices according to the mixture model. Hereafter the pseudo code of the Regularized Spectral EM algorithm:

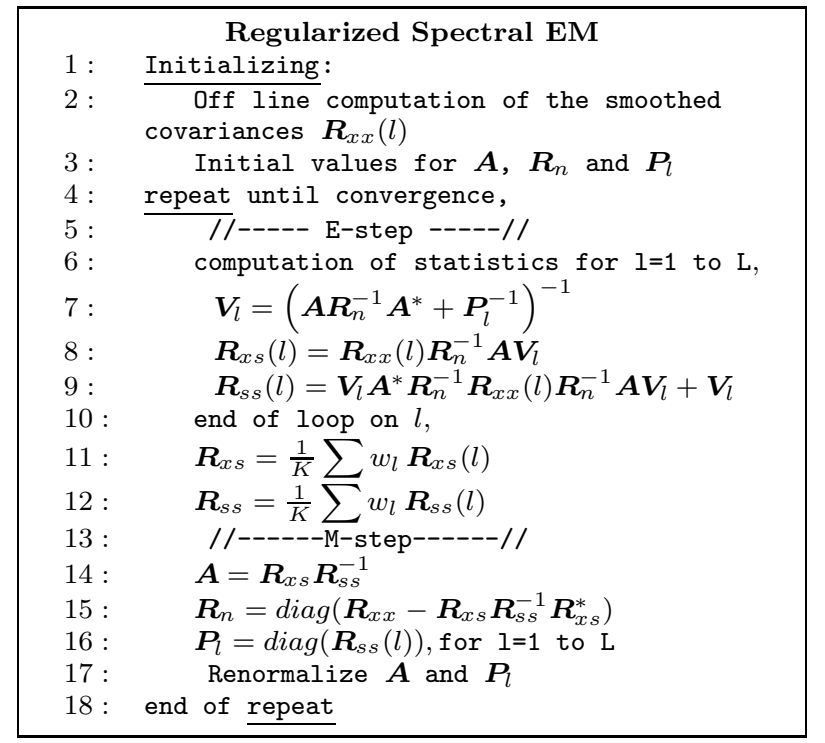

\section{ILLUSTRATION ON FMRI DATA}

The Regularized Spectral EM algorithm was applied to separate the time courses of fMRI data acquired at the FM Kirby Center for Functional Brain Imaging. The experiment consisted of presenting two periodic visual stimulus, shifted by $20 \mathrm{~s}$ from one another, to the subject. The stimuli consisted of an 8-Hz reversing checkerboard pattern presented for $15 \mathrm{~s}$ in the right visual hemifield, followed by $5 \mathrm{~s}$ of an asterisk fixation, followed by $15 \mathrm{~s}$ of checkerboard presented to the left visual hemifield, followed by $20 \mathrm{~s}$ of an asterisk fixation. The $55 \mathrm{~s}$ set of events was repeated four times for a total of $220 \mathrm{~s}$. Scans were acquired on a Philips NT 1.5-Tesla scanner. A sagittal localizer scan was performed first, followed by a $T_{1}$-weighted anatomic scan $[$ repeat time $(T R)=500 \mathrm{~ms}$, echo time $(\mathrm{TE})=30 \mathrm{~ms}$, 
field of view $=24 \mathrm{~cm}$, matrix $=256 \times 256$, slice thickness $=5 \mathrm{~mm}$, gap $=0.5 \mathrm{~mm}$ ] consisting of 18 slices through the entire brain including most of the cerebellum. Next, we acquired functional scans over the same 18 slices consisting of a single-shot, echo-planar scan $(\mathrm{TR}=1 \mathrm{~s}, \mathrm{TE}=39 \mathrm{~ms}$, field of view $=24 \mathrm{~cm}$, matrix $=64 \times 64$, slice thickness $=$ $5 \mathrm{~mm}$, gap $=0.5 \mathrm{~mm}$, flip angle $=90$ degrees) obtained consistently over a 3 -min, 40-s period for a total of 220 scans.

Our method is tested on two different slices where we expect, in each, two different event related components corresponding to the alternating activation of the right and left visual cortex as a response to an alternating visual stimulus presented to the subject.

The results of the proposed algorithm applied to the first data set (slice 10) are shown in Figure 4, where we have plotted three recovered image sources (the three columns of the estimated mixing matrix $\hat{\boldsymbol{A}}$ ) within their corresponding estimated time courses. We note the ability of the algorithm to extract the sources which have a time course correlated with the stimulus. The first and third sources correspond to the alternative activations of the right and left visual cortex as expected from the conditions of the stimulus presented to the subject. However, the algorithm extracts also another source (the second one) which has a spectral density similar to the first two sources. This shows that fixing the number of sources by intuitive expectation based on the experiment paradigm leads to wrong results. The results shown in Figure 4 were obtained by varying the number of sources and then studying a posteriori the results after convergence of the EM algorithm. An automatic selection of the number of components is thus needed for a complete blind analysis of the fMRI data. Figure 5 illustrates the times courses of the right and left visual cortex regions. We note the periodicity of the time courses and their relative inter-delay (around $20 \mathrm{~s}$ ) corresponding to the inter-delay of the stimulus (the checkerboard pattern was presented alternatively to the right and left visual hemifields). Figure 6 shows the spectrograms and the regularized spectra of the estimated three times courses. For comparison purposes, we reported the separation results of a temporal ICA InfoMax algorithm on the same data set, in Figure 7 . We have fixed the number of sources to 3. The ICA algorithm fails to extract the third source from the two alternative task related sources identified with the EM algorithm, mixing them with a higher frequency component.

In Figure 8, the results are plotted for the another slice (slice 14) characterized by a higher noise level than the previous slice. We note the ability of the algorithm to recover the sources as well. For this slice, we have processed only 110 timepoints as the acquisition rate was divided by 2 in the same experiment conditions as the data acquisition for the slice 10. Figure 9 illustrates the estimated time courses. We note their correlation with the expected activations of the brain as a response to the presented stimulus. The time delay is about 10 timepoints corresponding to the $20 \mathrm{~s}$ of the shift between the alternative periodic right and left visual stimulus. The InfoMax ICA algorithm gives poor results essentially because of the presence of noise in the processed data. This is to be expected as, in the ICA solution, we assume a noiseless observation model.

\section{Conclusion And Perspectives}

We have presented an EM algorithm to deal with real data suffering from non stationarity and a lack of enough points for spectral analysis. The separation method is essentially based on the diversity of the source smoothed periodograms. The non stationarity of second order statistics allows the mixing matrix identification without resorting to higher order statistics. The use of second order statistics (in other words, the Gaussian modeling) leads to an efficient and fast implementation of the EM algorithm. In fMRI data analysis, we have exploited this diversity between the time course spectra. The spatial pixel distributions represent the columns of the mixing matrix. Therefore, the Regularized Spectral EM algorithm allows a blind joint estimation of the brain source images within the spectra of their time courses. The event related sources are easily distinguished by the signature of the stimulus in their time course spectra.

\section{ACKNOWLEDGMENT}

This work was supported in part by the National Institutes of Health under grant 1 R 01 EB 000840-01 (to VC).

\section{REFERENCES}

[1] A. Hyvarinen and E. Oja, "Independent component analysis: Algorithms and applications", Neural Networks, vol. 13, pp. 411-430, 2000.

[2] A. Cichocki and S. Amari, Adaptive Blind Signal and Image Processing: Learning Algorithms and Applications, John Wiley, 2003.

[3] C. Jutten and J. Herault, "Blind separation of sources .1. an adaptive algorithm based on neuromimetic architecture", Signal Processing, vol. 24, no. 1, pp. 1-10, 1991.

[4] P. Comon, "Independent Component Analysis, a new concept ?", Signal processing, Special issue on Higher-Order Statistics, Elsevier, vol. 36 (3), pp. 287-314, April 1994.

[5] A. Hyvärinen and E. Oja, "A fast fixed-point algorithm for independent component analysis", Neural Computation, vol. 9(7), pp. 1483-1492, 1997.

[6] J.-F. Cardoso and A. Souloumiac, "Blind beamforming for non Gaussian signals", IEE Proceedings-F, vol. 140, no. 6, pp. 362370, December 1993.

[7] A. J. Bell and T. J. Sejnowski, "An information maximization approach to blind separation and blind deconvolution", Neural Computation, vol. 7, no. 6, pp. 1129-1159, 1995.

[8] E. Moulines, J. Cardoso, and E. Gassiat, "Maximum likelihood for blind separation and deconvolution of noisy signals using mixture models", in ICASSP-97, Munich, Germany, April 1997.

[9] H. Attias, "Blind separation of noisy mixture: An EM algorithm for independent factor analysis", Neural Computation, vol. 11, pp. 803-851, 1999.

[10] H. Snoussi and A. Mohammad-Djafari, "Fast joint separation and segmentation of mixed images", Journal of Electronic Imaging, vol. 13, no. 2, pp. 349-361, April 2004.

[11] A. Belouchrani, K. Abed Meraim, J.-F. Cardoso, and Éric Moulines, "A blind source separation technique based on second order statistics", IEEE Trans. Signal Processing, vol. 45, no. 2, pp. 434-44, February 1997.

[12] K. Rahbar and J. Reilly, "Blind source separation of convolved sources by joint approximate diagonalization of cross-spectral density matrices", in Proc. ICASSP, 2001.

[13] A. Belouchrani and M. Amin, "Blind source separation using time-frequency distributions: algorithm and asymptotic performance", in Proc. ICASSP, Munchen, 1997, pp. $3469-3472$. 
[14] K. Matsuoka, M. Ohya, and M. Kawamoto, "A neural net for blind separation of nonstationary sources", Neural Networks, vol. 8(3), pp. 411-419, 1995.

[15] S. Choi and A. Cichocki, "Blind separation of nonstationary sources in noisy mixtures", Electronics Letters, vol. 36(9), pp. 848-849, apr 2000.

[16] A. Souloumiac, "Blind source detection and separation using second order nonstationarity", in Proc. ICASSP, 1995, pp. 19121915.

[17] D.-T. Pham and J. Cardoso, "Blind separation of instantaneous mixtures of non stationary sources", IEEE Trans. Signal Processing, vol. 49, 9, no. 11, pp. 1837-1848, 2001.

[18] H. Snoussi and A. Mohammad-Djafari, "Penalized maximum likelihood for multivariate gaussian mixture", in Bayesian Inference and Maximum Entropy Methods, R. L. Fry, Ed. MaxEnt Workshops, August 2001, pp. 36-46, Amer. Inst. Physics.

[19] J. Cardoso, H. Snoussi, J. Delabrouille, and G. Patanchon, "Blind separation of noisy gaussian stationary sources. application to cosmic microwave background imaging", in Eusipco, Toulouse, France, September 2002.

[20] A. P. Dempster, N. M. Laird, and D. B. Rubin, "Maximum likelihood from incomplete data via the EM algorithm", $J . R$. Statist. Soc. B, vol. 39, pp. 1-38, 1977.

[21] F. Hlawatsch, G. Matz, H. Kirchauer, and W. Kozek, "Timefrequency formulation, design, and implementation of time varying optimal filters for signal estimation", IEEE Trans. Signal Processing, vol. 48, no. 5, pp. 1417-1432, May 2000.

[22] H. Snoussi, G. Patanchon, J. Macías-Pérez, A. MohammadDjafari, and J. Delabrouille, "Bayesian blind component separation for cosmic microwave background observations.", in Bayesian Inference and Maximum Entropy Methods, R. L. Fry, Ed. MaxEnt Workshops, August 2001, pp. 125-140, Amer. Inst. Physics.
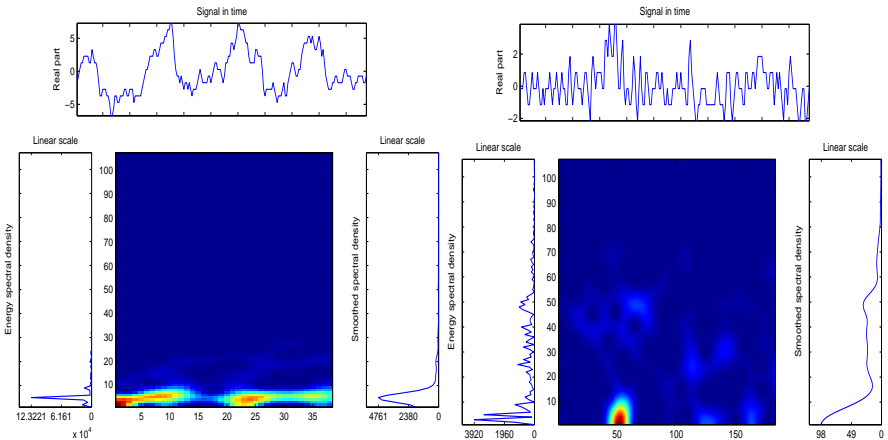

Fig. 3. Time frequency behavior of fMRI time courses: (a) fMRI time course of an event related activated region in the visual cortex, (b) non event related fMRI time course. The projection of STFT signal transform has a smoothness effect on the spectrum.

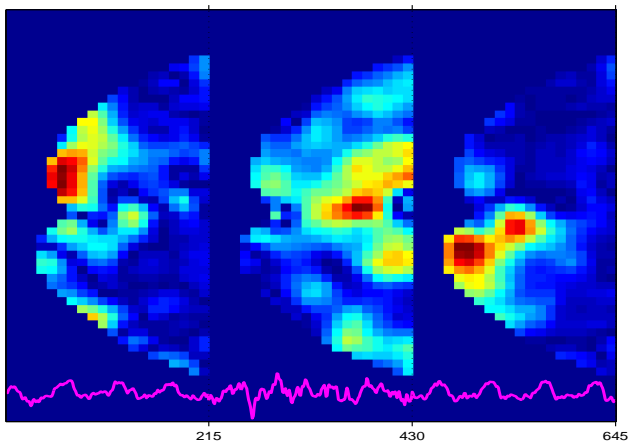

Fig. 4. The recovered sources with the Regularized Spectral EM algorithm for slice 10. The first and third sources correspond to the left and right visual cortex activations.

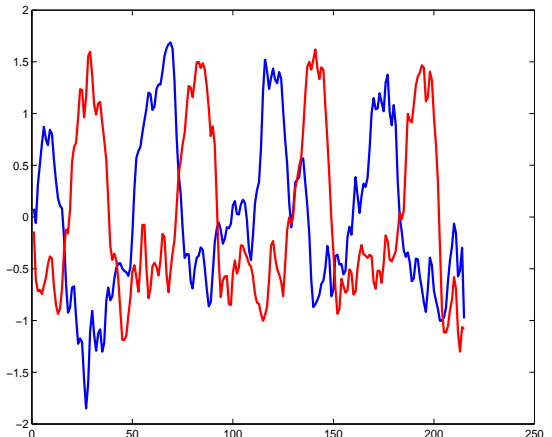

Fig. 5. The recovered time courses with the Regularized Spectral EM algorithm. Their temporal inter-delay is about $20 \mathrm{~s}$ corresponding to inter-delay between the alternative temporal stimulus presented to the subject.
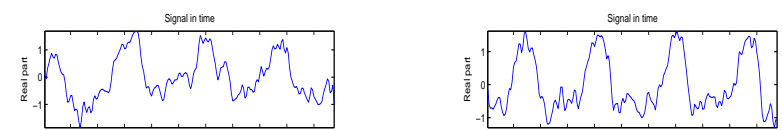

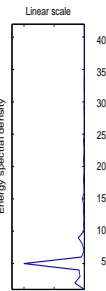
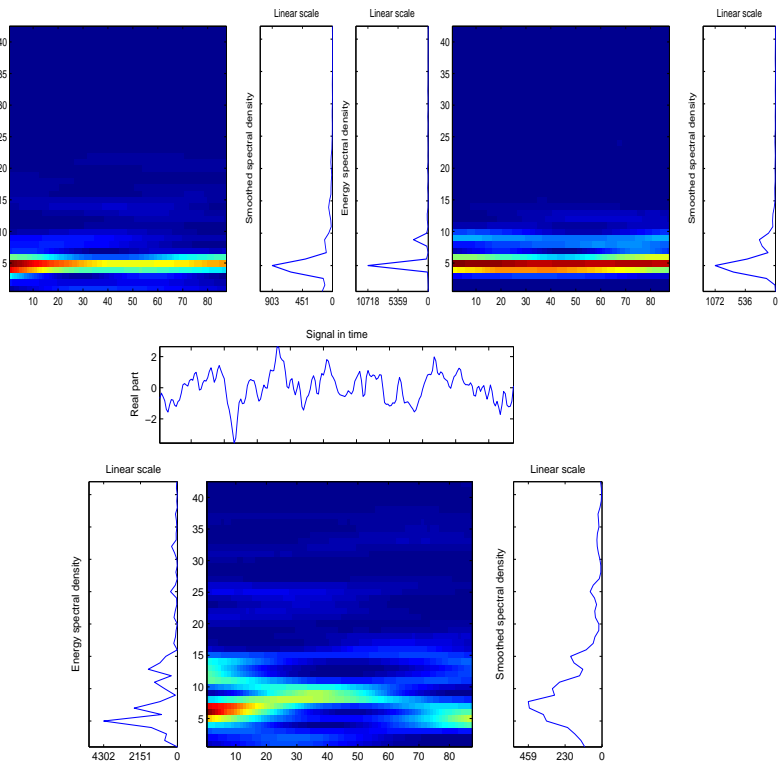

Fig. 6. The spectrograms of the estimated time courses: First, third and second according to the ordering in Figure 4.

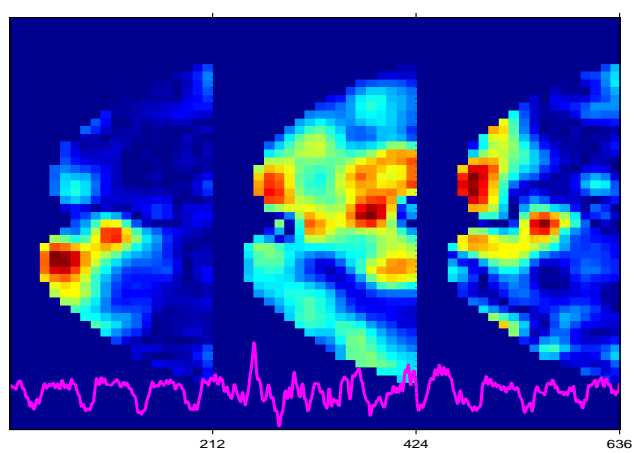

Fig. 7. The recovered sources with the ICA InfoMax algorithm for the Slice 10. The alternative task related sources are not separated from the transient signal (the image in the middle of the Figure 4). 


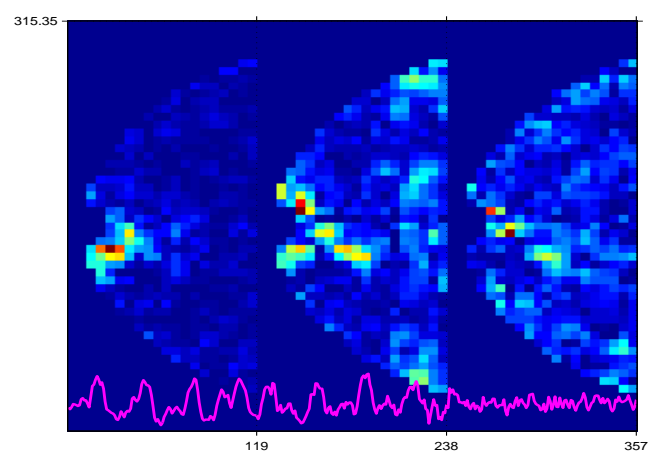

Fig. 8. The recovered sources with the Regularized Spectral EM algorithm for the slice 14. The first and second sources correspond to the right and left visual cortex.

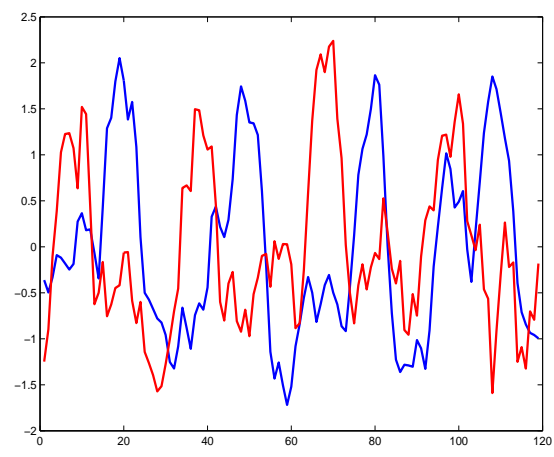

Fig. 9. The recovered time courses the Regularized Spectral EM algorithm. We note the periodicity and the inter-delay compatible with the periodicity and the inter-delay of the alternative left and right stimulus presented to the subject during scanning.

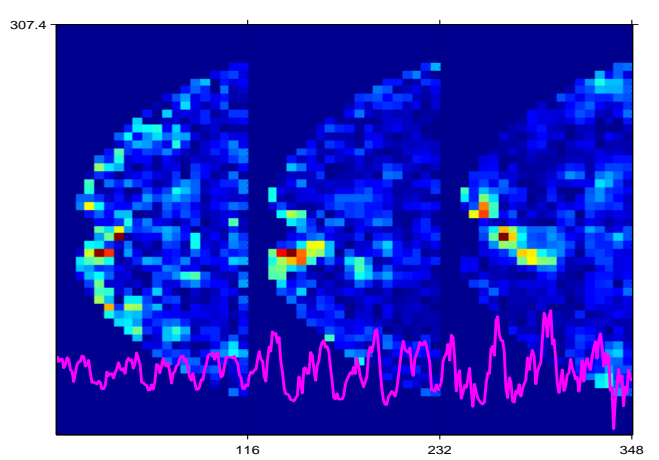

Fig. 10. The recovered sources with the ICA InfoMax algorithm for the slice 14 in a high noise environment. The sources are not identified. 